\title{
Impact of Perioperative Chemotherapy on Survival Outcomes Among Patients with Metastatic Colorectal Cancer to the Liver
}

Firas Baidoun ( $\nabla$ firasb2@hotmail.com )

Cleveland Clinic Foundation: Cleveland Clinic https://orcid.org/0000-0003-3584-9453

Zahi Merjaneh

Cleveland Clinic Foundation: Cleveland Clinic

Rama Nanah

Cleveland Clinic Foundation: Cleveland Clinic

Anas M. Saad

Cleveland Clinic Foundation: Cleveland Clinic

Omar Abdel-Rahman

University of Alberta Department of Oncology

\section{Research Article}

Keywords: chemotherapy, colorectal, liver, overall survival (OS)

Posted Date: May 27th, 2021

DOI: https://doi.org/10.21203/rs.3.rs-410003/v1

License: (c) (1) This work is licensed under a Creative Commons Attribution 4.0 International License. Read Full License 


\section{Abstract}

\section{Introduction}

While surgical resection for primary tumor and distant metastases is recommended among individuals with resectable colorectal liver metastases, the role and timing of perioperative systemic therapy is not yet defined.

\section{Materials and Methods}

The National Cancer Database was queried for patients diagnosed with metastatic colorectal adenocarcinoma with isolated liver metastases between 2004 and 2016. We evaluated the overall survival (OS) based on the perioperative chemotherapy status using Kaplan-Meier estimates and multivariable cox regression analyses.

\section{Results}

A total of 6,883 patients with metastatic CRC and liver metastases were included, within which 6,042 (87.8\%) were treated with surgery and chemotherapy, and $841(12.2 \%)$ were treated with surgery only. We found that patients who were treated with surgery and chemotherapy had better OS compared to patients who were treated with surgery only (median OS 42.9 months vs 6.4 months, $\mathrm{P}<0.001$ ). On multivariable analysis, chemotherapy was associated with better OS (HR 0.30; 95\% Cl: $0.275-0.328 ; \mathrm{P}<0.001)$.

We also found that patients who received multiagent chemotherapy had better OS compared to patients who received single-agent chemotherapy (median OS 43.7 months vs 36.1 months, $P<0.001$ ), patients who received neoadjuvant chemotherapy had better OS compared to patients who received adjuvant chemotherapy (median OS 48.6 months vs 38.6 months, $\mathrm{P}<0.001$ ).

\section{Conclusion}

Patients with CRC with isolated liver metastases who were treated with perioperative chemotherapy had significant improvement in OS compared to patients who didn't receive chemotherapy. Also, neoadjuvant chemotherapy had better OS compared to adjuvant chemotherapy, and multiagent chemotherapy had better OS compared to single-agent chemotherapy.

\section{Article Highlights:}

- Colorectal cancer (CRC) is the third most commonly diagnosed malignancy and the second leading cause of cancer-related death in the US.

- About $50 \%$ of CRC cases develop liver metastases.

- The survival benefit of perioperative chemotherapy in patients with metastatic CRC with liver metastases is still unclear.

- We found that perioperative chemotherapy improves the OS in metastatic CRC with liver metastases. 
- In metastatic CRC with liver metastases neoadjuvant and multiagent chemotherapy improve the OS compared to adjuvant and single-agent chemotherapy, respectively.

\section{Introduction}

Colorectal cancer (CRC) is the third most commonly diagnosed malignancy in the United States and the world and the second leading cause of cancer death in both sexes in 2020 in the United states ${ }^{1-3}$. Because the majority of the intestinal mesenteric drainage enters the hepatic portal venous system, hepatic metastases develop in approximately half of all colorectal cancer cases with nearly one-fifth to one-quarter of newly diagnosed metastatic colorectal cancer patients present with liver metastases at the time of primary diagnosis ${ }^{2,4}$. Surgical resection alone is the cornerstone of treatment for patients with liver metastases from colorectal cancer in whom there is at least a one in six chance of cure after hepatectomy and apparent clinical cure is achieved in those who survive 10 years ${ }^{5-7}$. In the past $15-20$ years, Profound improvements in outcomes of metastatic CRC resulted in more than a doubling of the 5year survival ${ }^{4,8,9}$. Although new treatment options have doubled overall survival (OS) for advanced disease, survival is still best for those with non-metastatic disease ${ }^{10}$. While the role of surgical resection is established in metastatic disease to the liver, the potential benefit from peri-operative chemotherapy remains unclear ${ }^{6,11}$. While some studies have shown that perioperative chemotherapy with surgery reduced the risk of progression-free survival events at 3-years in patients with resectable liver metastases $^{12}$, others concluded that preoperative chemotherapy did not prolong survival with colorectal cancer and resectable or marginally resectable liver metastasis ${ }^{13}$. Also, there's a lack of data in assessing the impact of timing of perioperative chemotherapy on survival (pre- versus post-operative), as well as in comparing the effect of single-agent vs multiagent chemotherapy on survival. We studied the survival outcomes of patients with CRC with liver metastases who underwent surgical management alone vs combined surgery and chemotherapy based on a real-world cohort. In addition to that, we assessed the effect of timing of chemotherapy on survival among patients who received chemotherapy and compared the OS between patients who received single-agent and patients who received multiagent chemotherapy as the first line of chemotherapy

\section{Patients And Methods}

\subsection{Data source}

The National Cancer Database (NCDB) is a nationwide database supported by the Commission on Cancer ( $\mathrm{CoC}$ ) of the American College of Surgeons and the American Cancer Society. About $70 \%$ of all cancer cases diagnosed in the United States from more than 1,500 cancer centers are included in this database. Details of patients' demographics, malignancy staging, and histological characteristics in addition to treatment and outcome information are provided in this database ${ }^{14}$. The CoC's NCDB and the hospitals participating in the CoC's NCDB are the sources of the de-identified data used herein; they have 
not verified and are not responsible for the statistical validity of the data analysis or the conclusions derived by the authors of the current study.

After we obtained the approval on our proposed protocol and the letter of support from the cancer committee chair at Cleveland Clinic, the NCDB was queried for patients diagnosed with metastatic colorectal adenocarcinoma between 2004 and 2016. Survival follow-up for the studied cohort is available till December 2016. It's worth mentioning that the metastatic status provided in the database is related to the status at the time of the diagnosis. NCDB doesn't provide the details about the possible nonmetastatic cases that subsequently developed metastatic disease.

\subsection{Patient selection}

For our study, we selected the patients who were diagnosed with metastatic colorectal adenocarcinoma with liver metastases at age of 18 or older. We excluded patients with metastases to other sites other than the liver. Patients who didn't have radical surgical resection to the primary tumor and/or didn't have surgery to distant metastatic sites were also excluded in addition to patients with unknown chemotherapy status. Also, we excluded patients who lost follow up which are defined by patients with missing details about their last contact status as alive or dead, and those with missing information about their time from diagnosis to the last contact.

\subsection{Variables}

Using the NCDB, we included the following patient demographic variables: Age, gender, race, median income, education level, insurance status, facility type (which includes community cancer program, comprehensive community cancer program, academic/research program, and integrated network cancer program) in addition to the geographic area which was classified as metropolitan, urban or rural location. Also, we collected the following disease-related variables: clinical tumor and nodal categories based on American Joint Committee on Cancer (AJCC) staging, microsatellite instability (MSI) status, CharlsonDeyo comorbidity score, and chemotherapy status.

\subsection{Outcome}

The primary outcome of this study was overall survival (OS) in months defined as the time from diagnosis to the time of death for any reason.

\subsection{Statistical analysis}

We compared the baseline demographics and characteristics between patients who were treated with surgery and chemotherapy versus patients who were treated with surgery only using the Pearson Chisquare test.

For OS comparisons, we split the cohort into two separate groups according to the type of treatment the patients received, the first group who was treated with surgery and chemotherapy and the second group who was treated with surgery only. Then, we compared the OS between the two groups. Moreover, we conducted additional OS comparisons (limited to patients who received perioperative chemotherapy) 
between patients who received adjuvant chemotherapy versus neoadjuvant chemotherapy; and between patients who received single-agent versus multiagent chemotherapy.

OS analyses were evaluated using the Kaplan-Meier survival method. A log-rank test was used to evaluate survival differences between groups. We used Cox regression analysis to conduct multivariable analyses to evaluate the factors associated with improved OS; hazard ratios (HRs) with associated 95\% confidence intervals $(\mathrm{Cl})$ were accordingly generated, statistical significance was defined as a $P$ value less than 0.05 for all analyses. The following factors were included in the multivariable model: facility type, age, gender, race, median household income, education level, insurance status, area, Charlson score, clinical T stage, clinical N stage, MSI status, and chemotherapy use.

All the above statistical analyses were performed using the SPSS Statistics 27.0

To further reduce any potential biases related to the variation in baseline characteristics between the patient group that received chemotherapy and the one that didn't receive chemotherapy, we used nearest neighbor, 1:1, propensity score matching using RStudio Version 1.3.1093 (MA, USA).

As a sensitivity analysis, we repeated all the above analyses after excluding patients who died within 90 days of the most definitive primary site surgery (to eliminate the effect of post-surgical complicationrelated mortality on overall survival).

To further examine the impact of MSI status on the above survival analyses, we repeated the above survival analyses among patients with MSI-H versus those with MSI-L status.

\section{Results}

\subsection{Baseline characteristics}

Figure-1 provides a flowchart for patient selection within the current study. We ended up with 6,883 patients with metastatic CRC and liver metastases; 6,042 (87.8\%) were treated with surgery and chemotherapy and $841(12.2 \%)$ were treated with surgery only. Compared to the surgery-only group, the perioperative group was likely to be younger ( 58 vs 70 years old), males ( $55.5 \%$ vs $47.4 \%$ ), and had more MSI-L status ( $27.8 \%$ vs $16.6 \%$ ). The baseline characteristics' comparison between the two groups is summarized in Table 1.

Table 1

Baseline characteristics of metastatic CRC patients with liver metastases according to the treatment modality as found in the National Cancer Database between 2010 and 2016 


\begin{tabular}{|c|c|c|c|c|}
\hline Characteristics & $\begin{array}{l}\text { All cases } \\
(\mathrm{N}=6,883)\end{array}$ & $\begin{array}{l}\text { Surgery } \\
\text { only } \\
(\mathrm{N}=841) \\
(12.2 \%)\end{array}$ & $\begin{array}{l}\text { Surgery and } \\
\text { chemotherapy } \\
(\mathrm{N}=6,042)(87.8 \%)\end{array}$ & $\begin{array}{l}P \\
\text { value } \\
\text { and } \\
\text { test }\end{array}$ \\
\hline Mean age in years & $59.54 \pm 13.05$ & $\begin{array}{l}70.28 \pm \\
12.92\end{array}$ & $58.04 \pm 12.35$ & $\begin{array}{l}<0.001 \\
\text { T-test }\end{array}$ \\
\hline Sex & & & & $\begin{array}{l}<0.001 \text {; } \\
\text { Chi- } \\
\text { square }\end{array}$ \\
\hline Female & $3,130(45.5 \%)$ & $\begin{array}{l}442 \\
(52.6 \%)\end{array}$ & $2,688(44.5 \%)$ & \\
\hline Male & 3,753 (54.5\%) & $\begin{array}{l}399 \\
(47.4 \%)\end{array}$ & $3,354(55.5 \%)$ & \\
\hline Race & & & & $\begin{array}{l}0.062 \\
\text { Chi- } \\
\text { square }\end{array}$ \\
\hline White & $5,682(82.6 \%)$ & $\begin{array}{l}672 \\
(79.9 \%)\end{array}$ & $5,010(82.9 \%)$ & \\
\hline African American & $826(12.0 \%)$ & $\begin{array}{l}124 \\
(14.7 \%)\end{array}$ & $702(11.6 \%)$ & \\
\hline Others & $329(4.8 \%)$ & $38(4.5 \%)$ & $291(4.8 \%)$ & \\
\hline Unknown & $46(0.7 \%)$ & $7(0.8 \%)$ & $39(0.6 \%)$ & \\
\hline Facility type & & & & $\begin{array}{l}<0.001 ; \\
\text { Chi- } \\
\text { square }\end{array}$ \\
\hline Community cancer program & $443(6.4 \%)$ & $87(10.3 \%)$ & $356(5.9 \%)$ & \\
\hline $\begin{array}{l}\text { Comprehensive Community Cancer } \\
\text { Program }\end{array}$ & $2,015(29.3 \%)$ & $\begin{array}{l}361 \\
(42.9 \%)\end{array}$ & $1,654(27.4 \%)$ & \\
\hline Academic/Research Program & $3,158(45.9 \%)$ & $\begin{array}{l}244 \\
(29.0 \%)\end{array}$ & $2,914(48.2 \%)$ & \\
\hline Integrated Network Cancer Program & $835(12.1 \%)$ & $\begin{array}{l}134 \\
(15.9 \%)\end{array}$ & $701(11.6 \%)$ & \\
\hline Unknown & $432(6.3 \%)$ & $15(1.8 \%)$ & $417(6.9 \%)$ & \\
\hline $\begin{array}{l}\text { Median household income 2012- } \\
2016\end{array}$ & & & & $\begin{array}{l}<0.001 ; \\
\text { Chi- } \\
\text { square }\end{array}$ \\
\hline Less than $\$ 40,227$ & $1,188(17.3 \%)$ & $\begin{array}{l}186 \\
(22.1 \%)\end{array}$ & $1,002(16.6 \%)$ & \\
\hline$\$ 40,227-\$ 50,353$ & $1,501(21.8 \%)$ & $\begin{array}{l}199 \\
(23.7 \%)\end{array}$ & $1,302(21.5 \%)$ & \\
\hline
\end{tabular}




\begin{tabular}{|c|c|c|c|c|}
\hline$\$ 50,354-\$ 63,332$ & $1,567(22.8 \%)$ & $\begin{array}{l}193 \\
(22.9 \%)\end{array}$ & $1,374(22.7 \%)$ & \\
\hline$\$ 63,333+$ & $2,539(36.9 \%)$ & $\begin{array}{l}254 \\
(30.2 \%)\end{array}$ & $2,285(37.8 \%)$ & \\
\hline Unknown & $88(1.3 \%)$ & $9(1.1 \%)$ & 79 (1.3\%) & \\
\hline $\begin{array}{l}\text { Education level } 2012-2016 \\
\text { (percentage of not graduated from } \\
\text { high school) }\end{array}$ & & & & $\begin{array}{l}<0.001 \\
\text { Chi- } \\
\text { square }\end{array}$ \\
\hline $17.6 \%$ or more & $1,237(18.0 \%)$ & $\begin{array}{l}217 \\
(25.8 \%)\end{array}$ & $1,020(16.9 \%)$ & \\
\hline $10.9 \%-17.5 \%$ & $1,803(26.2 \%)$ & $\begin{array}{l}246 \\
(29.3 \%)\end{array}$ & $1,557(25.8 \%)$ & \\
\hline $6.3 \%-10.8 \%$ & 2,017 (29.3\%) & $\begin{array}{l}202 \\
(24.0 \%)\end{array}$ & $1,815(30.0 \%)$ & \\
\hline Less than $6.3 \%$ & 1,753 (25.5\%) & $\begin{array}{l}171 \\
(20.3 \%)\end{array}$ & 1,582 (26.2\%) & \\
\hline Unknown & $73(1.1 \%)$ & $5(0.6 \%)$ & $68(1.1 \%)$ & \\
\hline Insurance status & & & & $\begin{array}{l}0.384 ; \\
\text { Chi- } \\
\text { square }\end{array}$ \\
\hline No & $225(3.3 \%)$ & $34(4.0 \%)$ & $191(3.2 \%)$ & \\
\hline Yes & $6,567(95.4 \%)$ & $\begin{array}{l}795 \\
(94.5 \%)\end{array}$ & $5,772(95.5 \%)$ & \\
\hline Unknown & $91(1.3 \%)$ & $12(1.4 \%)$ & $79(1.3 \%)$ & \\
\hline Area & & & & $\begin{array}{l}0.237 \\
\text { Chi- } \\
\text { square }\end{array}$ \\
\hline Metro counties & $5,545(80.6 \%)$ & $\begin{array}{l}697 \\
(82.9 \%)\end{array}$ & $4,848(80.2 \%)$ & \\
\hline Rural counties & $137(2.0 \%)$ & $17(2.0 \%)$ & $120(2.0 \%)$ & \\
\hline Urban counties & $1,031(15.0 \%)$ & $\begin{array}{l}112 \\
(13.3 \%)\end{array}$ & $919(15.2 \%)$ & \\
\hline Unknown & $170(2.5 \%)$ & $15(1.8 \%)$ & $155(2.6 \%)$ & \\
\hline Clinical T stage & & & & $\begin{array}{l}<0.001 \\
\text { Chi- } \\
\text { square }\end{array}$ \\
\hline T0 & $18(0.3 \%)$ & $1(0.1 \%)$ & $17(0.3 \%)$ & \\
\hline T1 & $316(4.6 \%)$ & $36(4.3 \%)$ & $280(4.6 \%)$ & \\
\hline
\end{tabular}




\begin{tabular}{|c|c|c|c|c|}
\hline $\mathrm{T} 2$ & $208(3.0 \%)$ & $17(2.0 \%)$ & $191(3.2 \%)$ & \\
\hline T3 & 1,617 (23.5\%) & $\begin{array}{l}135 \\
(16.1 \%)\end{array}$ & $1,482(24.5 \%)$ & \\
\hline T4 & $621(9.0 \%)$ & $\begin{array}{l}115 \\
(13.7 \%)\end{array}$ & $506(8.4 \%)$ & \\
\hline Tis & $15(0.2 \%)$ & $0(0.0 \%)$ & $15(0.2 \%)$ & \\
\hline Unknown & 4,088 (59.4\%) & $\begin{array}{l}537 \\
(63.9 \%)\end{array}$ & $3,551(58.8 \%)$ & \\
\hline Clinical N stage & & & & $\begin{array}{l}<0.001 \text {; } \\
\text { Chi- } \\
\text { square }\end{array}$ \\
\hline NO & 2,732 (39.7\%) & $\begin{array}{l}329 \\
(39.1 \%)\end{array}$ & $2,403(39.8 \%)$ & \\
\hline N1 & $1,694(24.6 \%)$ & $\begin{array}{l}166 \\
(19.7 \%)\end{array}$ & $1,528(25.3 \%)$ & \\
\hline N2 & $684(9.9 \%)$ & $98(11.7 \%)$ & $586(9.7 \%)$ & \\
\hline Unknown & $1,773(25.8 \%)$ & $\begin{array}{l}248 \\
(29.5 \%)\end{array}$ & $1,525(25.2 \%)$ & \\
\hline MSI status & & & & $\begin{array}{l}<0.001 \text {; } \\
\text { Chi- } \\
\text { square }\end{array}$ \\
\hline MSI-H & $208(3.0 \%)$ & $30(3.6 \%)$ & $178(2.9 \%)$ & \\
\hline MSI-L & $1,818(26.4 \%)$ & $\begin{array}{l}140 \\
(16.6 \%)\end{array}$ & $1,678(27.8 \%)$ & \\
\hline Unknown & 4,857 (70.6\%) & $\begin{array}{l}671 \\
(79.8 \%)\end{array}$ & $4,186(69.3 \%)$ & \\
\hline Charlson-Deyo score & & & & $\begin{array}{l}<0.001 \text {; } \\
\text { Chi- } \\
\text { square }\end{array}$ \\
\hline 0 & $5,325(77.4 \%)$ & $\begin{array}{l}523 \\
(62.2 \%)\end{array}$ & $4,802(79.5 \%)$ & \\
\hline 1 & 1,207 (17.5\%) & $\begin{array}{l}220 \\
(26.2 \%)\end{array}$ & $987(16.3 \%)$ & \\
\hline 2 & $258(3.7 \%)$ & $71(8.4 \%)$ & $187(3.1 \%)$ & \\
\hline 3 & $93(1.4 \%)$ & 27 (3.2\%) & $66(1.1 \%)$ & \\
\hline
\end{tabular}


Next, in our sensitivity analysis, we excluded all patients who died within 90 days of most definitive primary site surgery $(N=550)$, which left us with 6,333 patients; $5,834(92.1 \%)$ who were treated with surgery and chemotherapy, and 499 (7.9\%) who were treated with surgery only. The baseline characteristics' comparison between the two groups is summarized in Table 2.

Table 2

Baseline characteristics of metastatic CRC patients with liver metastases according to the treatment modality after excluding patients with 90 -day mortality of most definitive primary site surgery as found in the National Cancer Database between 2010 and 2016 


\begin{tabular}{|c|c|c|c|c|}
\hline Characteristics & $\begin{array}{l}\text { All cases } \\
(\mathrm{N}=6,333)\end{array}$ & $\begin{array}{l}\text { Surgery } \\
\text { only } \\
(\mathrm{N}=499) \\
(7.9 \%)\end{array}$ & $\begin{array}{l}\text { Surgery and } \\
\text { chemotherapy } \\
(\mathrm{N}=5,834)(92.1 \%)\end{array}$ & $\begin{array}{l}P \\
\text { value } \\
\text { and } \\
\text { test }\end{array}$ \\
\hline Mean age in years & $58.90 \pm 12.83$ & $\begin{array}{l}69.47 \pm \\
13.34\end{array}$ & $57.99 \pm 12.38$ & $\begin{array}{l}<0.001 \\
\text { T-test }\end{array}$ \\
\hline Sex & & & & $\begin{array}{l}<0.001 \text {; } \\
\text { Chi- } \\
\text { square }\end{array}$ \\
\hline Female & $2,882(45.5 \%)$ & $\begin{array}{l}274 \\
(54.9 \%)\end{array}$ & $2,608(44.7 \%)$ & \\
\hline Male & $3,451(54.5 \%)$ & $\begin{array}{l}225 \\
(45.1 \%)\end{array}$ & $3,226(55.3 \%)$ & \\
\hline Race & & & & $\begin{array}{l}0.256 \\
\text { Chi- } \\
\text { square }\end{array}$ \\
\hline White & $5,242(82.8 \%)$ & $\begin{array}{l}401 \\
(80.4 \%)\end{array}$ & $4,841(83.0 \%)$ & \\
\hline African American & $743(11.7 \%)$ & $68(13.6 \%)$ & $675(11.6 \%)$ & \\
\hline Others & $304(4.8 \%)$ & $24(4.8 \%)$ & $280(4.8 \%)$ & \\
\hline Unknown & $44(0.7 \%)$ & $6(1.2 \%)$ & $38(0.7 \%)$ & \\
\hline Facility type & & & & $\begin{array}{l}<0.001 \text {; } \\
\text { Chi- } \\
\text { square }\end{array}$ \\
\hline Community cancer program & $387(6.1 \%)$ & $42(8.4 \%)$ & $345(5.9 \%)$ & \\
\hline $\begin{array}{l}\text { Comprehensive Community Cancer } \\
\text { Program }\end{array}$ & $1,798(28.4 \%)$ & $\begin{array}{l}200 \\
(40.1 \%)\end{array}$ & $1,598(27.4 \%)$ & \\
\hline Academic/Research Program & $\begin{array}{l}2,987(47.2 \\
\%)\end{array}$ & $\begin{array}{l}171 \\
(34.3 \%)\end{array}$ & $2,816(48.3 \%)$ & \\
\hline Integrated Network Cancer Program & $742(11.7 \%)$ & $73(14.6 \%)$ & $669(11.5 \%)$ & \\
\hline Unknown & $419(6.6 \%)$ & $13(2.6 \%)$ & $406(7.0 \%)$ & \\
\hline $\begin{array}{l}\text { Median household income 2012- } \\
2016\end{array}$ & & & & $\begin{array}{l}0.002 \\
\text { Chi- } \\
\text { square }\end{array}$ \\
\hline Less than $\$ 40,227$ & $1,073(16.9 \%)$ & $\begin{array}{l}105 \\
(21.0 \%)\end{array}$ & $968(16.6 \%)$ & \\
\hline$\$ 40,227-\$ 50,353$ & $1,377(21.7 \%)$ & $\begin{array}{l}122 \\
(24.4 \%)\end{array}$ & $1,255(21.5 \%)$ & \\
\hline$\$ 50,354-\$ 63,332$ & $1,439(22.7 \%)$ & 119 & $1,320(22.6 \%)$ & \\
\hline
\end{tabular}


$(23.8 \%)$

\begin{tabular}{llll} 
\$63,333+ & $2,362(37.3 \%)$ & $\begin{array}{l}147 \\
(29.5 \%)\end{array}$ & $2,215(38.0 \%)$ \\
\hline Unknown & $82(1.3 \%)$ & $6(1.2 \%)$ & $76(1.3 \%)$
\end{tabular}

Education level 2012-2016 (percentage of not graduated from high school)

$<0.001$;

Chisquare

\begin{tabular}{llll}
\hline $17.6 \%$ or more & $1,116(17.6 \%)$ & $\begin{array}{l}128 \\
(25.7 \%)\end{array}$ & $988(16.9 \%)$ \\
\hline $10.9 \%-17.5 \%$ & $1,646(26.0 \%)$ & $\begin{array}{l}145 \\
(29.1 \%)\end{array}$ & $1,501(25.7 \%)$ \\
\hline $6.3 \%-10.8 \%$ & $1,866(29.5 \%)$ & $\begin{array}{l}117 \\
(23.4 \%)\end{array}$ & $1,749(30.0 \%)$ \\
\hline Less than $6.3 \%$ & $1,637(25.8 \%)$ & $\begin{array}{l}106 \\
(21.2 \%)\end{array}$ & $1,531(26.2 \%)$ \\
\hline Unknown & $68(1.1 \%)$ & $3(0.6 \%)$ & $65(1.1 \%)$
\end{tabular}

Insurance status

0.581 ;

Chi-

square

No

$200(3.2 \%) \quad 19(3.8 \%) \quad 181(3.1 \%)$

Yes

$6,049(95.5 \%) \quad 472$

(94.6\%)

$5,577(95.6 \%)$

$84(1.3 \%)$

$8(1.6 \%)$

$76(1.3 \%)$

Unknown

Area

(5)

Metro counties

$5,095(80.5 \%)$

411

$(82.4 \%)$

Rural counties

Urban counties

Unknown

Clinical T stage

\begin{tabular}{lll}
$129(2.0 \%)$ & $12(2.4 \%)$ & $117(2.0 \%)$ \\
\hline $952(15.0 \%)$ & $67(13.4 \%)$ & $885(15.2 \%)$ \\
\hline $157(2.5 \%)$ & $9(1.8 \%)$ & $148(2.5 \%)$
\end{tabular}

0.002;

Chi-

square

\begin{tabular}{llll} 
T0 & $14(0.2 \%)$ & $0(0.0 \%)$ & $14(0.2 \%)$ \\
\hline T1 & $298(4.7 \%)$ & $25(5.0 \%)$ & $273(4.7 \%)$ \\
\hline T2 & $199(3.1 \%)$ & $14(2.8 \%)$ & $185(3.2 \%)$
\end{tabular}




\begin{tabular}{|c|c|c|c|c|}
\hline T3 & $1,516(23.9 \%)$ & $86(17.2 \%)$ & $1,430(24.5 \%)$ & \\
\hline $\mathrm{T} 4$ & $540(8.5 \%)$ & $59(11.8 \%)$ & $481(8.2 \%)$ & \\
\hline Tis & $13(0.2 \%)$ & $0(0.0 \%)$ & $13(0.2 \%)$ & \\
\hline Unknown & $3,753(59.3 \%)$ & $\begin{array}{l}315 \\
(63.1 \%)\end{array}$ & $3,438(58.9 \%)$ & \\
\hline Clinical N stage & & & & $\begin{array}{l}0.008 \\
\text { Chi- } \\
\text { square }\end{array}$ \\
\hline NO & $2,535(40.0 \%)$ & $\begin{array}{l}219 \\
(43.9 \%)\end{array}$ & $2,316(39.7 \%)$ & \\
\hline N1 & $1,577(24.9 \%)$ & $\begin{array}{l}100 \\
(20.0 \%)\end{array}$ & $1,477(25.3 \%)$ & \\
\hline N2 & 607 (9.6\%) & $37(7.4 \%)$ & 570 (9.8\%) & \\
\hline Unknown & $1,614(25.5 \%)$ & $\begin{array}{l}143 \\
(28.7 \%)\end{array}$ & $1,471(25.2 \%)$ & \\
\hline MSI status & & & & $\begin{array}{l}<0.001 \text {; } \\
\text { Chi- } \\
\text { square }\end{array}$ \\
\hline MSI-H & $192(3.0 \%)$ & $20(4.0 \%)$ & $172(2.9 \%)$ & \\
\hline MSI-L & $1,718(27.1 \%)$ & $92(18.4 \%)$ & $1,626(27.9 \%)$ & \\
\hline Unknown & $4,423(69.8 \%)$ & $\begin{array}{l}387 \\
(77.6 \%)\end{array}$ & $4,036(69.2 \%)$ & \\
\hline Charlson-Deyo score & & & & $\begin{array}{l}<0.001 \text {; } \\
\text { Chi- } \\
\text { square }\end{array}$ \\
\hline 0 & $5,325(77.4 \%)$ & $\begin{array}{l}523 \\
(62.2 \%)\end{array}$ & $4,802(79.5 \%)$ & \\
\hline 1 & $1,207(17.5 \%)$ & $\begin{array}{l}220 \\
(26.2 \%)\end{array}$ & $987(16.3 \%)$ & \\
\hline 2 & $258(3.7 \%)$ & $71(8.4 \%)$ & $187(3.1 \%)$ & \\
\hline 3 & $93(1.4 \%)$ & 27 (3.2\%) & $66(1.1 \%)$ & \\
\hline
\end{tabular}

\subsection{Survival outcome}


We compared the OS between patients who were treated with surgery and chemotherapy versus patients who were treated with surgery only and found that patients who were treated with surgery and chemotherapy had better OS compared to patients who were treated with surgery only (median OS 42.9 months vs 6.4 months, $\mathrm{P}<0.001$ ) as illustrated in the Kaplan-Meier survival curves (Fig. 2).

Propensity score matching yielded 1,682 patients for analysis: 841 patients in the no chemotherapy group and 841 patients in the chemotherapy group (supplementary figure A shows the histogram for raw data and matched data). Comparison of baseline characteristics between the groups after propensity score matching is shown in supplementary table A.

We compared the OS between patients who received chemotherapy versus patients who didn't receive chemotherapy using Kaplan-Meier analysis on the propensity score-matched groups and found that patients who received chemotherapy had better OS than those who didn't receive chemotherapy as illustrated in the Kaplan-Meier survival curves $(P<0.001)$ (supplementary figure $A$ ).

\subsubsection{Excluding patients who died within 90 days of most definitive primary site surgery}

After excluding patients who died within 90 days of the most definitive primary site surgery, we compared the OS between patients who were treated with surgery and chemotherapy versus patients who were treated with surgery only. We found that patients who were treated with surgery and chemotherapy had better OS compared to patients who were treated with surgery only (median OS was 43.6 months vs 16.6 months, $\mathrm{P}<0.001$ ) as illustrated in the Kaplan-Meier survival curves (Fig. 3).

Propensity score matching yielded 998 patients for analysis: 499 patients in the no chemotherapy group and 499 patients in the chemotherapy group (supplementary figure B shows the histogram for raw data and matched data). Baseline characteristics comparison between the groups after propensity score matching is shown in supplementary table B.

We compared the OS between patients who received chemotherapy versus patients who didn't receive chemotherapy using Kaplan-Meier analysis on the propensity score-matched groups and found that patients who received chemotherapy had better OS than those who didn't receive chemotherapy as illustrated in the Kaplan-Meier survival curves $(P<0.001)$ (supplementary figure $B$ ).

\subsubsection{Subgroup analysis among patients who received chemotherapy}

\subsubsection{Single vs Multiagent chemotherapy}

After including only patients who received chemotherapy and excluding patients with the unknown number of chemotherapy agents they received, we compared the OS between patients who received single-agent chemotherapy and patients who received multiagent chemotherapy. We found patients who received multiagent chemotherapy had statistically significant better OS compared to patients who 
received single-agent chemotherapy (median OS was 43.7 months vs 36.1 months, $\mathrm{P}<0.001$ ) as illustrated in the Kaplan-Meier survival curves (Fig. 4).

Propensity score matching yielded 958 patients for analysis: 479 patients received single-agent chemotherapy and 479 patients received multiagent chemotherapy. We compared the OS between patients who received single-agent chemotherapy and patients who received multiagent chemotherapy using Kaplan-Meier analysis on the propensity score-matched groups and found that patients who received multiagent chemotherapy had statistically significant better OS compared to patients who received single-agent chemotherapy as illustrated in the Kaplan-Meier survival curves $(P<0.001)$ (supplementary figure C).

\subsubsection{Adjuvant vs neoadjuvant chemotherapy}

After excluding patients who did not received chemotherapy and with unknown time duration between diagnosis and surgery and time duration between diagnosis and chemotherapy, we compared the OS between patients who received neoadjuvant chemotherapy versus patients who received adjuvant chemotherapy. We found patients were treated with neoadjuvant chemotherapy had statistically significant better OS compared to patients were treated with adjuvant chemotherapy (median OS was 48.6 months vs 38.6 months, $P<0.001$ ) as illustrated in the Kaplan-Meier survival curves (Fig. 5).

Propensity score matching yielded 4428 patients for analysis: 2214 patients received chemotherapy before surgery and 2214 patients received chemotherapy after surgery. We compared the OS between patients who received chemotherapy before surgery and patients who received chemotherapy after surgery using Kaplan-Meier analysis on the propensity score-matched groups and we found that patients who received chemotherapy before surgery had statistically significant better OS compared to patients who received chemotherapy after surgery as illustrated in the Kaplan-Meier survival curves $(P<0.001)$ (supplementary figure D).

\subsubsection{Subgroup analysis according to MSI status}

\subsection{Chemotherapy and surgery vs surgery only}

After excluding patients with unknown MSI status, we ended up with 2,026 metastatic CRC patients with liver metastases and known MSI status. We split the patients into two groups according to the MSI status: MIS-H group and MSI-L group, then we compared the OS between patients who were treated with surgery and chemotherapy versus patients who were treated with surgery only in each MSI group. We found that patients who were treated with surgery and chemotherapy had better OS compared to patients who were treated with surgery only in both MSI-H and MSI-L groups as illustrated in the Kaplan-Meier survival curves (supplementary figure $\mathrm{E}$ ).

\subsection{Single vs Multiagent chemotherapy}

After excluding patients with unknown MSI status, we ended up with 1,789 metastatic CRC patients with liver metastases and known MSI status who received chemotherapy with a known number of 
chemotherapy agents they received. We split the patients into two groups according to the MSI status: MIS-H group and MSI-L group, then we compared the OS between patients who received single-agent chemotherapy and patients who received multiagent chemotherapy in each MSI group. In the MSI-L group, we found patients who received multiagent chemotherapy had statistically significant better OS compared to patients who received single-agent chemotherapy. Whereas in the MSI-H group, there was no difference in OS between the two treatment groups (supplementary figure F).

\subsection{Adjuvant vs neoadjuvant chemotherapy}

After excluding patients with unknown MSI status, we ended up with 1,755 metastatic CRC patients with liver metastases and known MSI status who received chemotherapy with known time from diagnosis to surgery and time from diagnosis to chemotherapy. We split the patients into two groups according to the MSI status: MSI-H group and MSI-L group, then we compared the OS between patients who received chemotherapy before surgery and patients who received chemotherapy after surgery in each MSI group. In the MSI-L group, we found patients who received chemotherapy before surgery had statistically significant better OS compared to patients who received chemotherapy after surgery. Whereas in the MSI$\mathrm{H}$ group, there was no difference in OS between the two treatment groups (supplementary figure $\mathrm{G}$ ).

\subsection{Multivariable analysis}

Multivariable analysis was done to assess factors affecting OS. In the entire cohort, factors associated with worse OS were older age (HR 1.021; 95\% Cl 1.018-1.025, $\mathrm{P}<0.001)$, African American race compared to Caucasian race (HR 1.171; $95 \% \mathrm{Cl} 1.058-1.296, \mathrm{P}=0.002)$, not having insurance (HR 1.217; $95 \% \mathrm{Cl} 1.027-1.442, \mathrm{P}=0.024)$, clinical N1 and N2 stage compared to N0 stage (HR $1.224 ; 95 \% \mathrm{Cl}$ 1.122-1.335, $\mathrm{P}<0.001$ and HR 1.907; 95\% Cl 1.699-2.141, $\mathrm{P}<0.001$, respectively), community cancer program (HR for Academic/Research Program and Integrated Network Cancer Program compared to community cancer program was $0.625 ; 95 \% \mathrm{Cl} 0.549-0.712, \mathrm{P}<0.001$ and $\mathrm{HR} 0.842 ; 95 \% \mathrm{Cl} 0.726-$ $0.978, P=0.024$, respectively), higher Charlson-Deyo scores (HR for Charlson-Deyo scores 0 compared to the Charlson-Deyo score of 3 was $0.768 ; 95 \% \mathrm{Cl} 0.602-0.980, \mathrm{P}=0.034$ ), MSI-high (HR for MSI-stable compared to MSI-high was $0.818 ; 95 \% \mathrm{Cl} 0.677-0.988, \mathrm{P}=0.037$ ) and not receiving chemotherapy (HR for receiving chemotherapy compared to not receiving chemotherapy was $0.300 ; 95 \% \mathrm{Cl} 0.275-0.328, \mathrm{P}<$ 0.001) (Table 3).

Table 3

Multivariate analysis for metastatic CRC patients with liver metasteses 


\begin{tabular}{|c|c|c|c|}
\hline Variables & $\mathrm{HR}$ & $95 \% \mathrm{Cl}$ & $P$ value \\
\hline \multicolumn{4}{|l|}{ Facility type } \\
\hline Community cancer program & \multicolumn{3}{|c|}{ Reference } \\
\hline Comprehensive Community Cancer Program & 0.973 & $\begin{array}{l}0.853- \\
1.108\end{array}$ & 0.676 \\
\hline Academic/Research Program & 0.625 & $\begin{array}{l}0.549- \\
0.712\end{array}$ & $<0.001$ \\
\hline Integrated Network Cancer Program & 0.842 & $\begin{array}{l}0.726- \\
0.978\end{array}$ & 0.024 \\
\hline Unknown & 1.140 & $\begin{array}{l}0.931- \\
1.395\end{array}$ & 0.204 \\
\hline Age & 1.021 & $\begin{array}{l}1.018- \\
1.025\end{array}$ & $<0.001$ \\
\hline \multicolumn{4}{|l|}{ Gender } \\
\hline Female & 0.950 & $\begin{array}{l}0.890- \\
1.013\end{array}$ & 0.118 \\
\hline Male & \multicolumn{3}{|c|}{ Reference } \\
\hline \multicolumn{4}{|l|}{ Race } \\
\hline African American & 1.171 & $\begin{array}{l}1.058- \\
1.296\end{array}$ & 0.002 \\
\hline Others & 0.868 & $\begin{array}{l}0.734- \\
1.026\end{array}$ & 0.098 \\
\hline Unknown & 0.571 & $\begin{array}{l}0.363- \\
0.898\end{array}$ & 0.015 \\
\hline White & \multicolumn{3}{|c|}{ Reference } \\
\hline \multicolumn{4}{|l|}{ Median household income 2012-2016 } \\
\hline Less than $\$ 40,227$ & \multicolumn{3}{|c|}{ Reference } \\
\hline$\$ 40,227-\$ 50,353$ & 1.020 & $\begin{array}{l}0.917- \\
1.136\end{array}$ & 0.710 \\
\hline$\$ 50,354-\$ 63,332$ & 0.972 & $\begin{array}{l}0.867- \\
1.089\end{array}$ & 0.620 \\
\hline$\$ 63,333+$ & 0.892 & $\begin{array}{l}0.787- \\
1.011\end{array}$ & 0.074 \\
\hline Unknown & 0.604 & $\begin{array}{l}0.298- \\
1.226\end{array}$ & 0.163 \\
\hline
\end{tabular}


high school)

$17.6 \%$ or more

Reference

$10.9 \%-17.5 \%$

0.965

0.873 -

1.067

0.483

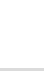

$6.3 \%-10.8 \%$

0.929

0.832 -

1.038

Less than $6.3 \%$

0.965

0.850 -

1.095

Unknown

1.384

0.634 -

3.022

0.192

0.579

0.415

\section{Insurance status}

No

1.217

1.027 -

1.442

0.024

0.740 -

1.303

0.900

Unknown

0.982

Yes

Reference

Area

Metro counties

Reference

Rural counties

1.020

0.814

1.276

Urban counties

1.027

0.934 -

1.131

Unknown

1.089

0.886 -

1.338

0.866

0.580

0.417

\section{Charlson score}

\begin{tabular}{|c|c|c|c|}
\hline 0 & 0.768 & $\begin{array}{l}0.602- \\
0.980\end{array}$ & 0.034 \\
\hline 1 & 0.830 & $\begin{array}{l}0.646- \\
1.067\end{array}$ & 0.145 \\
\hline 2 & 1.109 & $\begin{array}{l}0.837- \\
1.468\end{array}$ & 0.471 \\
\hline 3 & Refere & & \\
\hline \multicolumn{4}{|c|}{ Clinical T stage } \\
\hline TO & \multicolumn{3}{|c|}{ Reference } \\
\hline T1 & 0.591 & $\begin{array}{l}0.311 \text { - } \\
1.122\end{array}$ & 0.108 \\
\hline
\end{tabular}




\begin{tabular}{|c|c|c|c|}
\hline $\mathrm{T} 2$ & 0.473 & $\begin{array}{l}0.246- \\
0.911\end{array}$ & 0.025 \\
\hline T3 & 0.475 & $\begin{array}{l}0.253- \\
0.889\end{array}$ & 0.020 \\
\hline T4 & 0.739 & $\begin{array}{l}0.393- \\
1.390\end{array}$ & 0.348 \\
\hline Tis & 0.667 & $\begin{array}{l}0.262- \\
1.696\end{array}$ & 0.395 \\
\hline Unknown & 0.626 & $\begin{array}{l}0.335- \\
1.170\end{array}$ & 0.142 \\
\hline \multicolumn{4}{|c|}{ Clinical N stage } \\
\hline NO & \multicolumn{3}{|c|}{ Reference } \\
\hline $\mathrm{N} 1$ & 1.224 & $\begin{array}{l}1.122- \\
1.335\end{array}$ & $<0.001$ \\
\hline N2 & 1.907 & $\begin{array}{l}1.699- \\
2.141\end{array}$ & $<0.001$ \\
\hline Unknown & 1.325 & $\begin{array}{l}1.219- \\
1.440\end{array}$ & $<0.001$ \\
\hline \multicolumn{4}{|c|}{ MSI status } \\
\hline High & \multicolumn{3}{|c|}{ Reference } \\
\hline stable & 0.818 & $\begin{array}{l}0.677- \\
0.988\end{array}$ & 0.037 \\
\hline Unknown & 0.833 & $\begin{array}{l}0.695- \\
0.999\end{array}$ & 0.49 \\
\hline \multicolumn{4}{|c|}{ Chemotherapy } \\
\hline NO & \multicolumn{3}{|c|}{ Reference } \\
\hline Yes & 0.300 & $\begin{array}{l}0.275- \\
0.328\end{array}$ & $<0.001$ \\
\hline
\end{tabular}

In the sensitivity cohort (after excluding patients who died within 90 days of the most definitive primary site surgery), factors associated with worse OS were age (HR 1.021; 95\% Cl 1.018-1.025, $\mathrm{P}<0.001)$, African American race compared to white race (HR 1.179; 95\% Cl 1.057-1.315, $\mathrm{P}=0.003)$, community cancer program (HR for Academic/Research Program and Integrated Network Cancer Program compared to community cancer program was $0.664 ; 95 \% \mathrm{Cl} 0.576-0.766, \mathrm{P}<0.001$ and $\mathrm{HR} 0.830 ; 95 \% \mathrm{Cl} 0.704-$ $0.978, P=0.026$, respectively), clinical N1 and N2 stage compared to N0 stage (HR $1.206 ; 95 \% \mathrm{Cl} 1.100-$ 1.322, $\mathrm{P}<0.001$ and $\mathrm{HR} 1.809 ; 95 \% \mathrm{Cl} 1.597-2.050, \mathrm{P}<0.001$, respectively), and not receiving 
chemotherapy (HR for receiving chemotherapy compared to not receiving chemotherapy was $0.472 ; 95 \%$ Cl 0.422-0.529, $\mathrm{P}<0.001$ ) (Table 4).

\section{Table 4}

Multivariate analysis for metastatic CRC patients with liver metastases after excluding patients with 90day mortality of most definitive primary site surgery 


\begin{tabular}{|c|c|c|c|}
\hline Variables & HR & $95 \% \mathrm{Cl}$ & $P$ value \\
\hline \multicolumn{4}{|l|}{ Facility type } \\
\hline Community cancer program & \multicolumn{3}{|c|}{ Reference } \\
\hline Comprehensive Community Cancer Program & 1.012 & $\begin{array}{l}0.877- \\
1.169\end{array}$ & 0.867 \\
\hline Academic/Research Program & 0.664 & $\begin{array}{l}0.576- \\
0.766\end{array}$ & $<0.001$ \\
\hline Integrated Network Cancer Program & 0.830 & $\begin{array}{l}0.704- \\
0.978\end{array}$ & 0.026 \\
\hline Unknown & 1.184 & $\begin{array}{l}0.956- \\
1.466\end{array}$ & 0.121 \\
\hline Age & 1.021 & $\begin{array}{l}1.018 \text { - } \\
1.025\end{array}$ & $<0.001$ \\
\hline \multicolumn{4}{|l|}{ Gender } \\
\hline Female & 0.974 & $\begin{array}{l}0.910- \\
1.044\end{array}$ & 0.459 \\
\hline Male & \multicolumn{3}{|c|}{ Reference } \\
\hline \multicolumn{4}{|l|}{ Race } \\
\hline African American & 1.179 & $\begin{array}{l}1.057 \text { - } \\
1.315\end{array}$ & 0.003 \\
\hline Others & 0.890 & $\begin{array}{l}0.746- \\
1.061\end{array}$ & 0.194 \\
\hline Unknown & 0.572 & $\begin{array}{l}0.354- \\
0.923\end{array}$ & 0.022 \\
\hline White & \multicolumn{3}{|c|}{ Reference } \\
\hline \multicolumn{4}{|l|}{ Median household income 2012-2016 } \\
\hline Less than $\$ 40,227$ & \multicolumn{3}{|c|}{ Reference } \\
\hline$\$ 40,227-\$ 50,353$ & 1.017 & $\begin{array}{l}0.907- \\
1.139\end{array}$ & 0.778 \\
\hline$\$ 50,354-\$ 63,332$ & 0.961 & $\begin{array}{l}0.851- \\
1.085\end{array}$ & 0.521 \\
\hline$\$ 63,333+$ & 0.883 & $\begin{array}{l}0.773- \\
1.009\end{array}$ & 0.068 \\
\hline Unknown & 0.659 & $\begin{array}{l}0.310- \\
1.403\end{array}$ & 0.280 \\
\hline
\end{tabular}


high school)

$17.6 \%$ or more

Reference

$10.9 \%-17.5 \%$

0.967

0.868

1.077

0.540

$6.3 \%-10.8 \%$

0.927

0.824 1.043

Less than $6.3 \%$

0.949

0.829 1.087

Unknown

1.225

0.533 -

2.813

\section{Insurance status}

No

1.179

0.981

1.418

Unknown

1.007

0.747 1.357

Reference

Yes

Reference

Metro counties

1.037

0.821 -

1.311

0.758

Rural counties

1.028

0.929

1.138

Unknown

1.133

0.913 -

1.407

0.079

0.965

Urban counties

(1.2.

\section{Charlson score}

\begin{tabular}{|c|c|c|c|}
\hline 0 & 0.870 & $\begin{array}{l}0.656- \\
1.155\end{array}$ & 0.335 \\
\hline 1 & 0.928 & $\begin{array}{l}0.694- \\
1.240\end{array}$ & 0.613 \\
\hline 2 & 1.299 & $\begin{array}{l}0.940- \\
1.796\end{array}$ & 0.113 \\
\hline 3 & Refere & & \\
\hline \multicolumn{4}{|c|}{ Clinical T stage } \\
\hline T0 & \multicolumn{3}{|c|}{ Reference } \\
\hline T1 & 0.820 & $\begin{array}{l}0.383- \\
1.754\end{array}$ & 0.609 \\
\hline
\end{tabular}




\begin{tabular}{|llll|}
\hline T2 & 0.652 & $0.301-$ & 0.279 \\
\hline T4 & & 1.413 & \\
\hline Tis & 0.643 & $0.304-$ & 0.247 \\
& & 1.359 & \\
\hline Unknown & 0.981 & $0.462-$ & 0.960 \\
\hline Clinical N stage & & 2.083 & \\
\hline No & 0.844 & $0.295-$ & 0.752 \\
\hline N1 & & 2.416 & \\
\hline N2 & 0.836 & $0.397-$ & 0.638 \\
\hline Unknown & & 1.762 & \\
\hline MSI status & & & $<0.001$ \\
\hline High & Reference & & $<$ \\
\hline stable & 1.206 & $1.100-$ & \\
\hline Unknown & & 1.322 & \\
\hline Chemotherapy & 1.809 & $1.597-$ & $<0.001$ \\
\hline No & & 2.050 & \\
\hline Yes & 1.305 & $1.195-$ & $<0.001$ \\
\hline & & 1.427 & \\
\hline
\end{tabular}

\section{Discussion}

To our knowledge, this is the largest real-world study that looked at the chemotherapy effect in metastatic CRC with only liver metastases who were treated with metastasectomy and compared the OS between patients who received perioperative chemotherapy versus patients treated with surgery only in addition to 
comparing the OS between adjuvant chemotherapy and neoadjuvant chemotherapy and comparing the OS between single-agent and multiagent chemotherapy.

Our study showed that patients with metastatic CRC with liver metastases who were treated with chemotherapy in addition to surgery had statistically significant better OS compared to patients who didn't receive chemotherapy. Also, in the multivariable analysis, we found that chemotherapy was independently associated with better OS.

There is limited data about the role of perioperative chemotherapy in patients with liver metastases from CRC. Most of the studies had a small number of patients and were designed to evaluate the effect of chemotherapy on recurrence- and progression-free survival rather than overall survival. And the majority of the studies that evaluated the effect of chemotherapy on OS didn't report a significant difference in the OS between patients who received perioperative chemotherapy and patients who didn't.

Nordlinger et al. in a European Organization for Research and Treatment of Cancer (EORTC) trial based on 364 patients with metastatic CRC and up to four liver metastases, who were recruited from 78 hospitals from different European countries, compared the outcomes of 182 patients who received perioperative FOLFOX-4 chemotherapy to those of 182 patients who had surgery only. Initial results showed chemotherapy was associated with better 3-year progression-free survival (PFS) compared to surgical resection alone. After a median follow-up of 8.5 years, it was found that patients who received perioperative chemotherapy had a better median OS and 5-year OS, but it wasn't statistically significant. There're multiple factors in that study that could contribute to the lack of difference in the OS between the two groups. In addition to the small number of included patients, the study was designed with PFS being the primary endpoint whereas OS was a secondary endpoint. Also, the perioperative group had a higher number of non-cancer deaths compared to the surgery-only group ${ }^{12,15}$. Similarly, Portier et al. in the FFCD trial compared the outcomes between patients who had surgery only versus patients who had adjuvant chemotherapy with fluorouracil and folinic acid in 173 patients with liver metastases from CRC. They found that patients who received adjuvant chemotherapy had better 5-year OS, but it wasn't statistically significant. Several factors could be behind this result, the study wasn't designed with overall survival as an endpoint, also recurrences in both groups were treated by second-line chemotherapy, or by repeat liver resections, which influenced the natural history of the disease ${ }^{16}$. Hasegawa et al. in a randomized controlled trial, assigned 180 patients with colorectal cancer liver metastasis into two groups; patients who received adjuvant chemotherapy with uracil-tegafur and leucovorin and patients who had had surgery only. They found that patients who received adjuvant chemotherapy had significantly reduced recurrence compared to the patients who had surgery only, but there was no difference in the OS (66.1\% vs. $66.8 \%, P=0.409$ ). Multiple limitations also may have contributed to this result; the short period of follow-up (median follow-up was less than 5 years), a small number of included patients, and the way the study was designed with recurrence-free survival as the primary endpoint whereas the OS was a secondary endpoint ${ }^{17}$. Kemeny et al. did a randomized trial of 75 patients with one to three hepatic metastases of CRC where patients were randomized to hepatic resection alone or resection followed by hepatic arterial infusion of floxuridine and systemic 5-FU. The results showed that patients who received 
chemotherapy had a better 4-year OS and median OS, but the difference wasn't statistically significant ( $P$ $=0.6)^{18}$.

While the previously mentioned studies failed to show a significant effect of chemotherapy on OS, another study reported a positive effect of chemotherapy on OS. A pooled analysis of two trials done by Mitry et al. that included 278 patients with metastatic CRC (171 patients with liver metastases from the FFCD trial and 107 patients with liver or lung metastases from the ENG trial) and found that compared to patients who had surgery alone, patients who had adjuvant chemotherapy had better median PFS and median OS, but the differences were not statistically significant. Whereas, in multivariable analysis, they found that adjuvant chemotherapy was associated with better PFS and $0 S^{19}$ which aligns with our findings.

On the other hand, Kanemitsu et al. in a randomized trial of 300 patients with metastatic CRC with an unlimited number of liver metastases, found that patients who had adjuvant modified FOLFOX6 regimen had better disease-free survival, but a numerically worse 3-year OS and worse 5-year OS compared to patients who had hepatectomy alone, but the differences were not statistically significant ${ }^{20}$.

Among patients who received chemotherapy, we found that patients were treated with neoadjuvant chemotherapy had better OS compared to patients were treated with adjuvant chemotherapy. Also, we found patients who received multiagent chemotherapy had better OS compared to patients who received single-agent chemotherapy.

The vast majority of trials, that was done on metastatic CRC, assessed the effect of perioperative chemotherapy compared to surgical resection only, and while the comparison between adjuvant and neoadjuvant chemotherapy in non-metastatic CRC has been investigated, there are very few prospective studies done in metastatic cases ${ }^{21,22}$. While some studies suggest neoadjuvant chemotherapy is beneficial in unresectable disease and helps in improving the complete resection success rate ${ }^{23}$, other studies showed that neoadjuvant chemotherapy was associated with increased liver toxicity and suggest adjuvant chemotherapy was more beneficial in managing micro-metastases with less liver toxicity-related complications ${ }^{24-27}$. In the setting of not having a head-to-head comparison between adjuvant and neoadjuvant chemotherapy in metastatic disease, the NCCN guideline still recommends using perioperative chemotherapy for a total of 6 months without a preference for adjuvant or neoadjuvant chemotherapy ${ }^{28}$.

When it comes to the comparison between the single-agent and multiagent chemotherapy, few studies looked at that in the setting of metastatic disease. Seymour et al. evaluated 2135 patients with inoperable metastatic or locoregional CRC and compared the OS in 3 different chemotherapy strategies; the first group received single-agent as a first line and single-agent as a second line, the second group received single-agent as a first line and combination chemotherapy as a second line, the third group received combination chemotherapy as a first line. The study showed that the group treated with combination chemotherapy as a first line had better OS compared to the group that received single-agent 
chemotherapy in both first and second line. Interestingly, there was no statistically significant difference in the OS between the group that received single-agent chemotherapy as a first and second line and the group that received single-agent chemotherapy as a first line and combination chemotherapy as a second line. These results are similar to our result in one way that patients who received multiagent chemotherapy as a first line had better OS than patients who received single-agent as a first line. Unfortunately, the NCDB doesn't provide details about the second line chemotherapy to be able to compare patients according to second line chemotherapy like the mentioned study ${ }^{29}$. The biggest limiting factor in this study is that recruited patients were selected as a poor-prognosis group who were definitely incurable and patients with operable metastases were excluded.

Koopman et al. in phase III randomized controlled trial (CAIRO study), reported no statistically significant difference in the OS between patients with advanced CRC who received combination chemotherapy as a first line and patients who received the same chemotherapy agents but were given sequentially ${ }^{30}$. Similar to what we mentioned before, the chemotherapy details in NCDB are limited to first line chemotherapy.

Our study has some limiting factors that need to be discussed; first, the type of chemotherapy that patients received was not available. This could have impacted the outcomes of some patients receiving adjuvant chemotherapy. Second, the differentiation between single-agent and multiagent chemotherapy is limited to the first line of chemotherapy not total chemotherapy the patient may have received. Third, the distribution and completeness of surgical resection of liver metastases were not specified in the database. This is an important element that could have affected patients' outcomes if imbalanced between both groups. Fourth, NCDB also doesn't have details about other oncologic endpoints like disease-free survival and cancer-specific survival (both represent important endpoints to evaluate the effect of chemotherapy). Fifth, the retrospective nature of data collection within the NCDB might have affected the veracity of the analyses as well.

It's worth mentioning as well that in our study, patients who had surgery only were older compared to patients who received perioperative chemotherapy (mean age in years 70 vs 58 ) which could be a significant factor that affects the difference in the OS between the two groups as older patients tend to have more comorbidities and as a result more likely not to receive chemotherapy. To account for this potential bias, we did a propensity score matching for age and Charlson comorbidity score as well as multivariable Cox regression analysis incorporating other relevant baseline factors.

\section{Conclusion}

In conclusion, our study shows that the addition of chemotherapy to surgery improves the OS in metastatic CRC with liver metastases treated with metastasectomy. Also, neoadjuvant and multiagent chemotherapy seem to improve the OS compared to adjuvant and single-agent chemotherapy, respectively. Further large-scale studies are needed to evaluate the effect of chemotherapy role and timing not only on recurrence and PFS but also on OS. 


\section{Abbreviations}

AJCC: American Joint Committee on Cancer, Cl: confidence intervals, CoC: commission on cancer, CRC: colorectal cancer, CRC: colorectal cancer, MSI: microsatellite instability, NCDB: National Cancer Database, OS: overall survival, PFS: progression-free survival, US: United States

\section{Declarations}

\section{Disclosures:}

The authors have no conflict of interest to declare.

\section{Funding:}

This paper was not funded.

\section{Acknowledgment}

None.

\section{Author contributions:}

Firas Baidoun: data analysis, data interpretation, manuscript writing.

Zahi Merjaneh: manuscript writing.

Rama Nanah: manuscript writing.

Anas M. Saad: manuscript editing, critical revision.

Omar Abdel-Rahman: study concept, manuscript editing, critical revision.

\section{Acknowledgment}

None.

\section{Data Availability Statement}

The data we used in this study is available from the National Cancer Database.

\section{Consent}

Not required.

\section{Conflict of interest}

None 


\section{References}

1. Siegel RL, Miller KD, Jemal A. Cancer statistics. 2020. CA Cancer J Clin. 2020;70(1):7-30. doi:10.3322/caac.21590.

2. Zarour LR, Anand S, Billingsley KG, et al. Colorectal Cancer Liver Metastasis: Evolving Paradigms and Future Directions. Cmgh. 2017;3(2):163-73. doi:10.1016/j.jcmgh.2017.01.006.

3. Baidoun F, Elshiwy K, Elkeraie Y, et al. Colorectal Cancer Epidemiology: Recent trends and Impact on Outcomes. Curr Drug Targets. 2020;21. doi:10.2174/1389450121999201117115717.

4. Kanas GP, Taylor A, Primrose JN, et al. Survival after liver resection in metastatic colorectal cancer: Review and meta-analysis of prognostic factors. Clin Epidemiol. 2012;4(1):283-301. doi:10.2147/CLEP.S34285.

5. Abdalla EK, Vauthey JN, Ellis LM, et al. Recurrence and outcomes following hepatic resection, radiofrequency ablation, and combined resection/ablation for colorectal liver metastases. Ann Surg. 2004;239(6):818-27. doi:10.1097/01.sla.0000128305.90650.71.

6. Tomlinson JS, Jarnagin WR, DeMatteo RP, et al. Actual 10-year survival after resection of colorectal liver metastases defines cure. J Clin Oncol. 2007;25(29):4575-80. doi:10.1200/JC0.2007.11.0833.

7. Oweira H, Mehrabi A, Reissfelder C, Abdel-Rahman OA, Real-World. Population-Based Analysis of the Outcomes of Colorectal Cancer Patients with Isolated Synchronous Liver or Lung Metastases Treated with Metastasectomy. World J Surg. 2020;44(5):1604-11. doi:10.1007/s00268-019-05353-9.

8. Messersmith WA. NCCN Guidelines Updates: Management of Metastatic Colorectal Cancer. J Natl Compr Canc Netw. 2019;17(55):599-601. doi:10.6004/jnccn.2019.5014.

9. Chakedis J, Schmidt CR. Surgical Treatment of Metastatic Colorectal Cancer. Surg Oncol Clin N Am. 2018;27(2):377-99. doi:10.1016/j.soc.2017.11.010.

10. Dekker E, Tanis PJ, Vleugels JLA, Kasi PM, Wallace MB. Colorectal cancer. Lancet. 2019. doi:10.1016/S0140-6736(19)32319-0.

11. Fong Y, Fortner J, Sun RL, Brennan MF, Blumgart LH. Clinical Score for Predicting Recurrence After Hepatic Resection for Metastatic Colorectal Cancer. Ann Surg. 1999;230(3):309.

12. Nordlinger B, Sorbye H, Glimelius B, et al. Perioperative chemotherapy with FOLFOX4 and surgery versus surgery alone for resectable liver metastases from colorectal cancer (EORTC Intergroup trial 40983): a randomised controlled trial. Lancet. 2008;371(9617):1007-16. doi:10.1016/S01406736(08)60455-9.

13. Ichikawa N, Kamiyama T, Yokoo H, et al. Preoperative chemotherapy in colorectal cancer patients with synchronous liver metastasis. Mol Clin Oncol. 2020;12(4):374-83. doi:10.3892/mco.2020.1992.

14. National Cancer Database - About the National Cancer Database. Accessed on Septemeber 8th. 2020. https://www.facs.org/quality-programs/cancer/ncdb/about. 
15. Nordlinger B, Sorbye H, Glimelius B, et al. Perioperative FOLFOX4 chemotherapy and surgery versus surgery alone for resectable liver metastases from colorectal cancer (EORTC 40983): long-term results of a randomised, controlled, phase 3 trial. Lancet Oncol. 2013;14(12):1208-15. doi:10.1016/S1470-2045(13)70447-9.

16. Portier G, Elias D, Bouche O, et al. Multicenter Randomized Trial of Adjuvant Fluorouracil and Folinic Acid Compared With Surgery Alone After Resection of Colorectal Liver Metastases: FFCD ACHBTH AURC 9002 Trial. J Clin Oncol. 2006;24(31):4976-82. doi:10.1200/JC0.2006.06.8353.

17. Hasegawa K, Saiura A, Takayama T, et al Adjuvant Oral Uracil-Tegafur with Leucovorin for Colorectal Cancer Liver Metastases: A Randomized Controlled Trial. Su C-W, ed. PLoS One. 2016;11(9):e0162400. doi:10.1371/journal.pone.0162400.

18. Kemeny MM, Adak S, Gray B, et al. Combined-modality treatment for resectable metastatic colorectal carcinoma to the liver: Surgical resection of hepatic metastases in combination with continuous infusion of chemotherapy - An intergroup study. J Clin Oncol. 2002;20(6):1499-505. doi:10.1200/JC0.20.6.1499.

19. Mitry E, Fields ALA, Bleiberg H, et al. Adjuvant Chemotherapy After Potentially Curative Resection of Metastases From Colorectal Cancer: A Pooled Analysis of Two Randomized Trials. J Clin Oncol. 2008;26(30):4906-11. doi:10.1200/JC0.2008.17.3781.

20. Kanemitsu Y, Shimizu Y, Mizusawa J, et al. A randomized phase II/III trial comparing hepatectomy followed by mFOLFOX6 with hepatectomy alone for liver metastasis from colorectal cancer: JCOG0603 study. J Clin Oncol. 2020;38(15_suppl):4005-5. doi:10.1200/JCO.2020.38.15_suppl.4005.

21. Sauer R, Becker H, Hohenberger W, et al. Preoperative versus Postoperative Chemoradiotherapy for Rectal Cancer. N Engl J Med. 2004;351(17):1731-40. doi:10.1056/nejmoa040694.

22. De Gooyer JM, Verstegen MG, Lam-Boer J, et al. Neoadjuvant Chemotherapy for Locally Advanced T4 Colon Cancer: A Nationwide Propensity-Score Matched Cohort Analysis. Dig Surg. 2020;37(4):292-301. doi:10.1159/000503446.

23. Bismuth H, Adam R, Lévi F, et al. Resection of nonresectable liver metastases from colorectal cancer after neoadjuvant chemotherapy. Ann Surg. 1996;224(4):509-22. doi:10.1097/00000658$199610000-00009$.

24. Vauthey JN, Pawlik TM, Ribero D, et al. Chemotherapy regimen predicts steatohepatitis and an increase in 90-day mortality after surgery for hepatic colorectal metastases. J Clin Oncol. 2006;24(13):2065-72. doi:10.1200/JC0.2005.05.3074.

25. Mehta NN, Ravikumar R, Coldham CA, et al. Effect of preoperative chemotherapy on liver resection for colorectal liver metastases. Eur J Surg Oncol. 2008;34(7):782-6. doi:10.1016/j.ejso.2007.09.007.

26. Fernandez FG, Ritter J, Goodwin JW, Linehan DC, Hawkins WG, Strasberg SM. Effect of steatohepatitis associated with irinotecan or oxaliplatin pretreatment on resectability of hepatic colorectal metastases. J Am Coll Surg. 2005;200(6):845-53. doi:10.1016/j.jamcollsurg.2005.01.024. 
27. Parks R, Gonen M, Kemeny N, et al. Adjuvant Chemotherapy Improves Survival after Resection of Hepatic Colorectal Metastases: Analysis of Data from Two Continents. J Am Coll Surg. 2007;204(5):753-61. doi:10.1016/j.jamcollsurg.2006.12.036.

28. NCCN Clinical Practice Guidelines in. Oncology - Colon Cancer. https://www.nccn.org/professionals/physician_gls/pdf/colon.pdf.

29. Seymour MT, Maughan TS, Ledermann JA, et al. Different strategies of sequential and combination chemotherapy for patients with poor prognosis advanced colorectal cancer (MRC FOCUS): a randomised controlled trial. Lancet. 2007;370(9582):143-52. doi:10.1016/S0140-6736(07)61087-3.

30. Koopman M, Antonini NF, Douma J, et al. Sequential versus combination chemotherapy with capecitabine, irinotecan, and oxaliplatin in advanced colorectal cancer (CAIRO): a phase III randomised controlled trial. Lancet. 2007;370(9582):135-42. doi:10.1016/S0140-6736(07)61086-1.

\section{Figures}




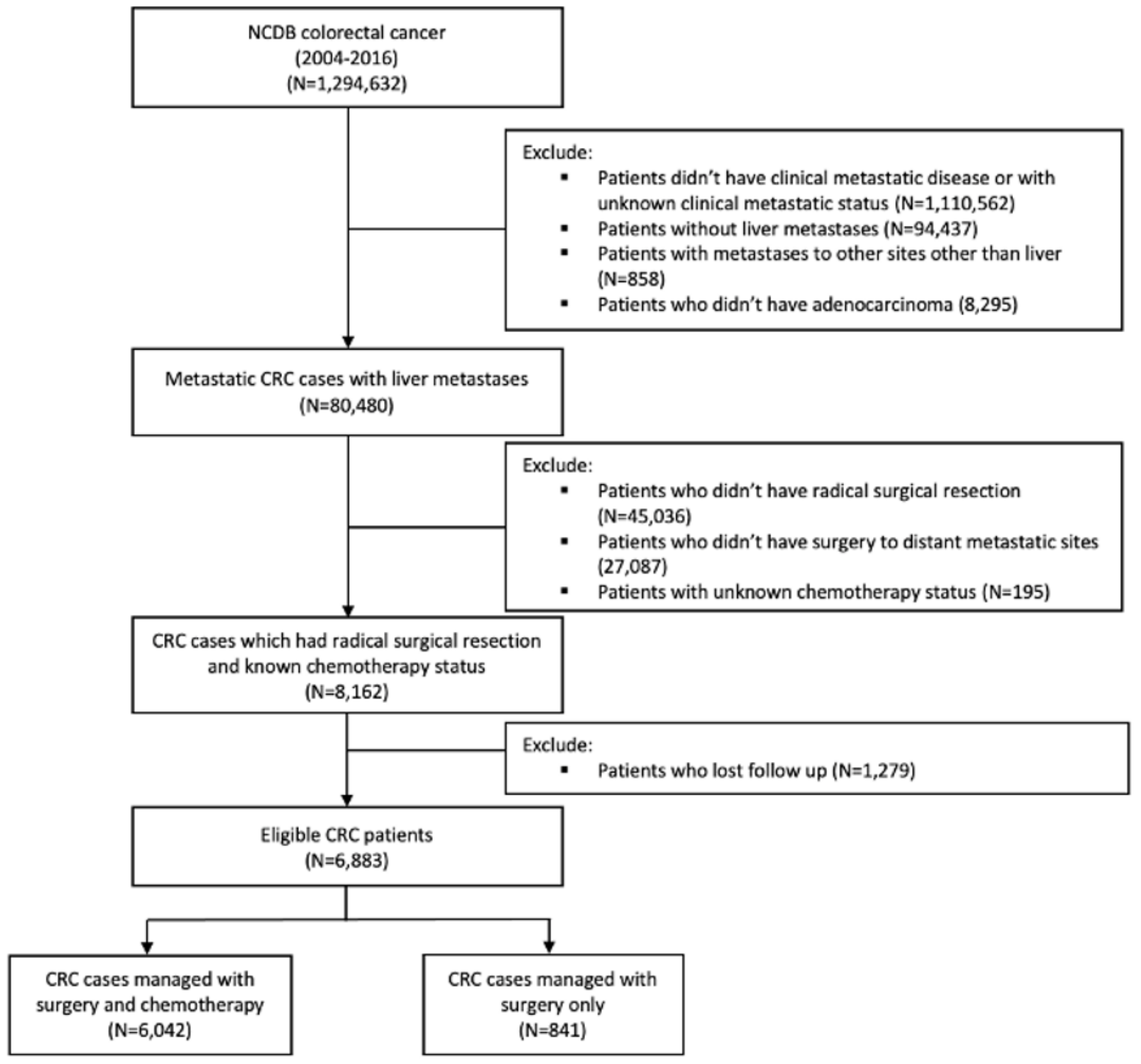

Figure 1

provides a flowchart for patient selection within the current study 


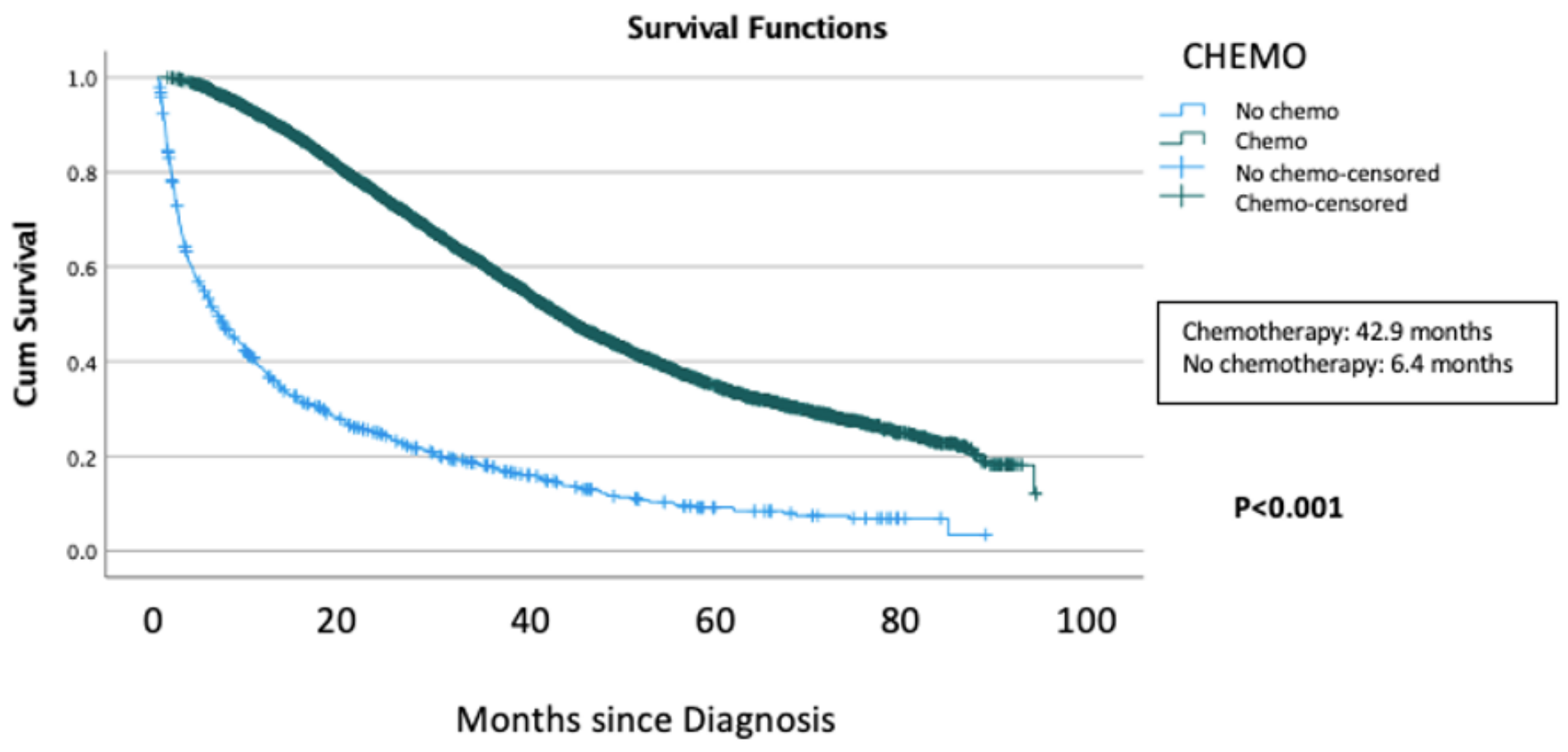

Figure 2

We compared the OS between patients who were treated with surgery and chemotherapy versus patients who were treated with surgery only and found that patients who were treated with surgery and chemotherapy had better OS compared to patients who were treated with surgery only (median OS 42.9 months vs 6.4 months, $\mathrm{P}<0.001$ ) as illustrated in the Kaplan-Meier survival curves
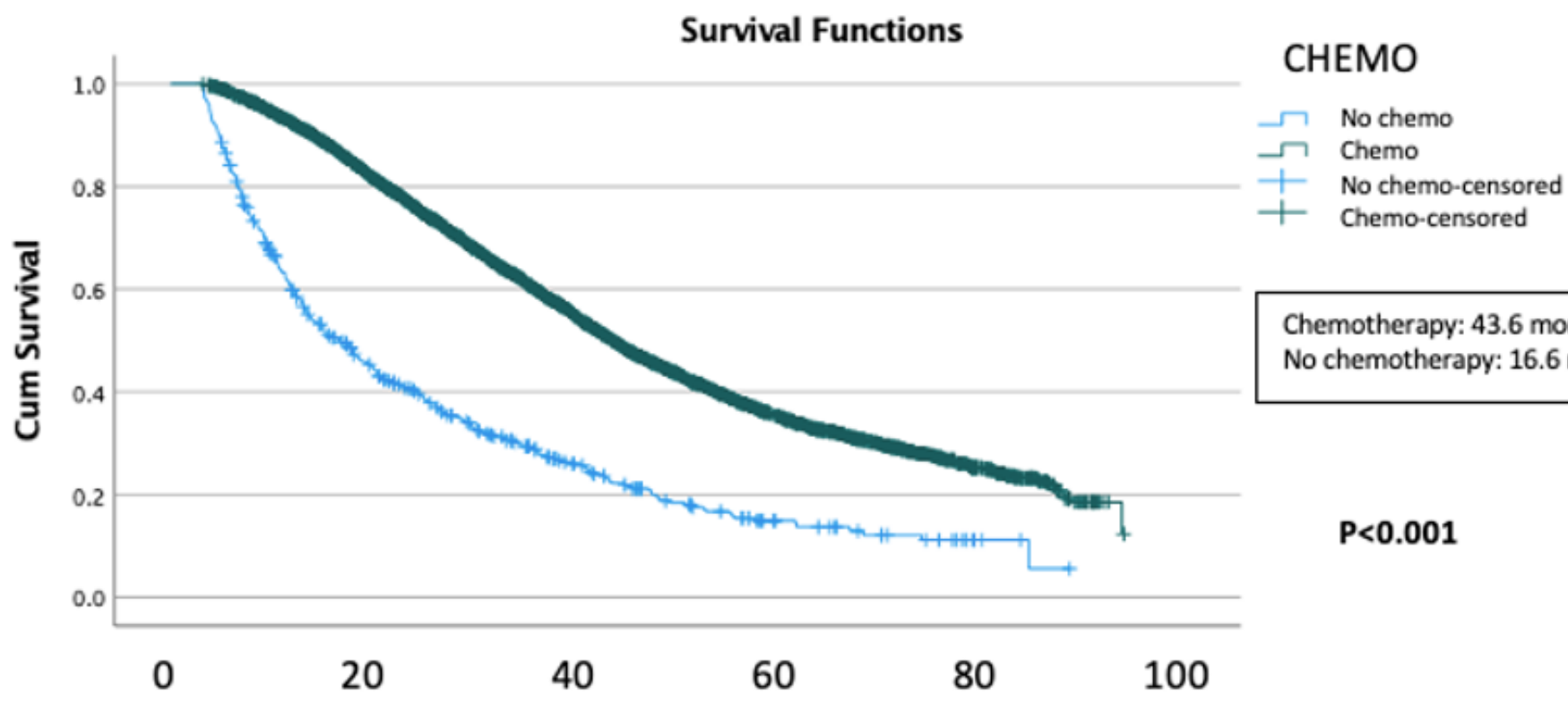

Chemotherapy: 43.6 months No chemotherapy: 16.6 months

Months since Diagnosis

Figure 3 
After excluding patients who died within 90 days of the most definitive primary site surgery, we compared the OS between patients who were treated with surgery and chemotherapy versus patients who were treated with surgery only. We found that patients who were treated with surgery and chemotherapy had better OS compared to patients who were treated with surgery only (median OS was 43.6 months vs 16.6 months, $\mathrm{P}<0.001)$ as illustrated in the Kaplan-Meier survival curves

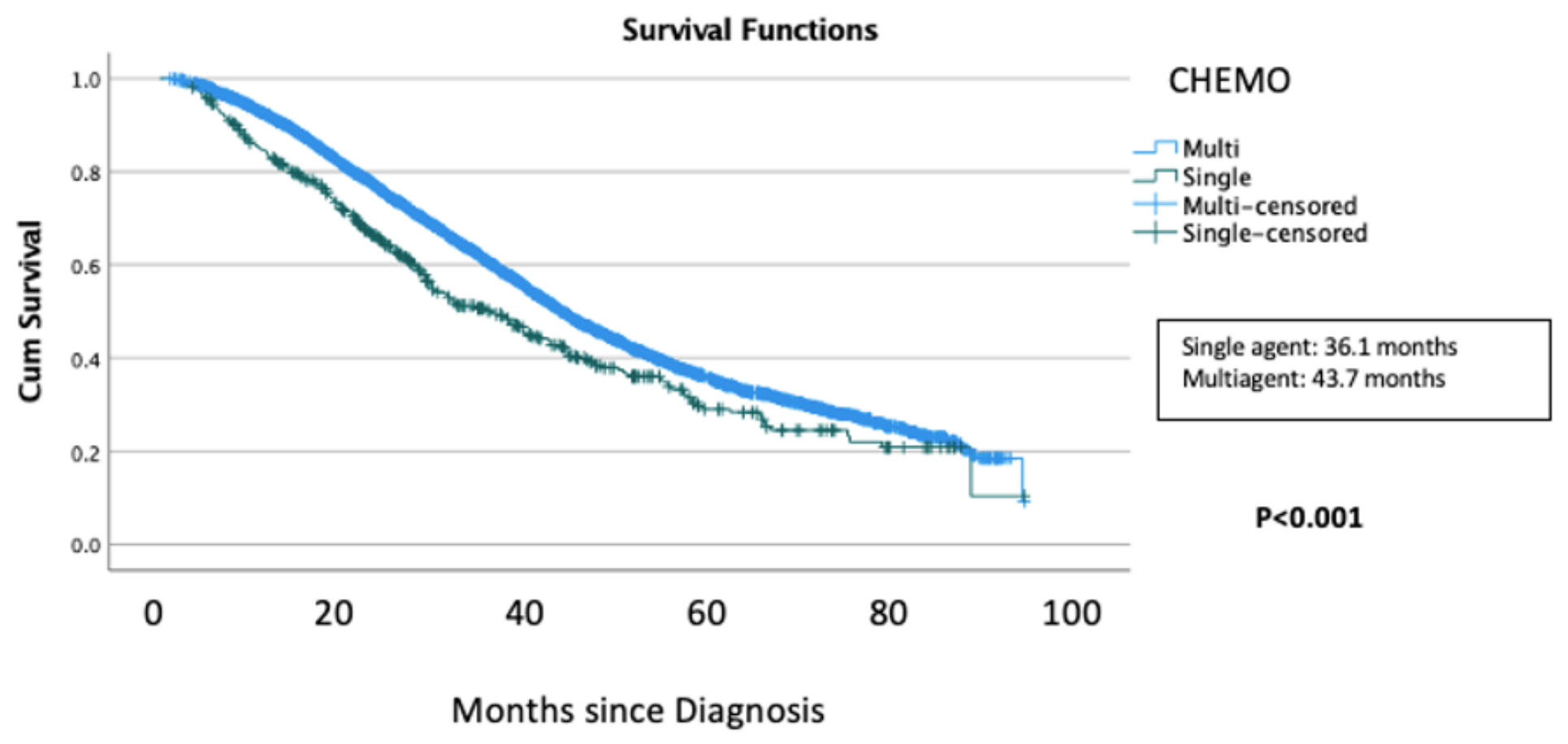

\section{Figure 4}

After including only patients who received chemotherapy and excluding patients with the unknown number of chemotherapy agents they received, we compared the OS between patients who received single-agent chemotherapy and patients who received multiagent chemotherapy. We found patients who received multiagent chemotherapy had statistically significant better OS compared to patients who received single-agent chemotherapy (median OS was 43.7 months vs 36.1 months, $P<0.001$ ) as illustrated in the Kaplan-Meier survival curves 


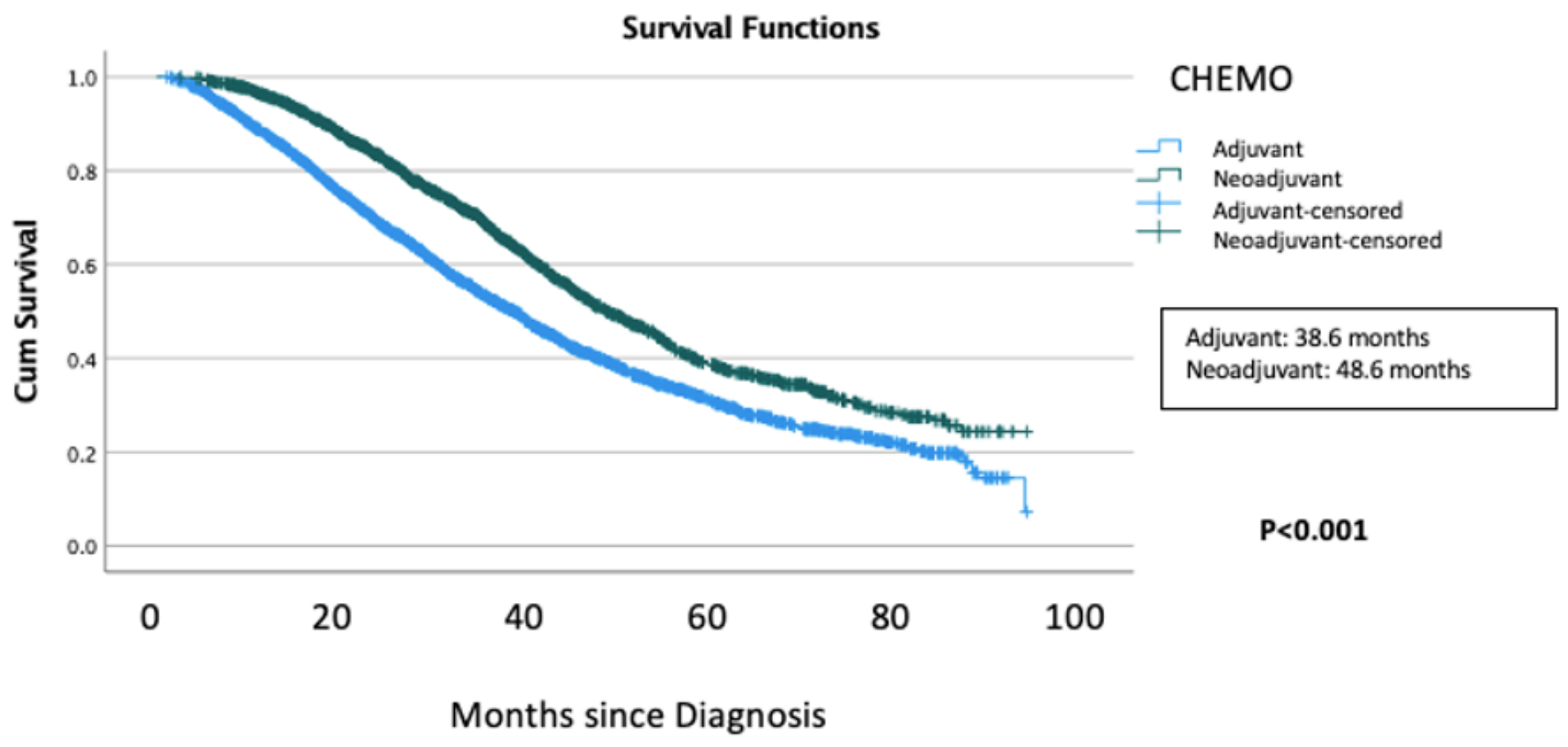

Figure 5

After excluding patients who did not received chemotherapy and with unknown time duration between diagnosis and surgery and time duration between diagnosis and chemotherapy, we compared the OS between patients who received neoadjuvant chemotherapy versus patients who received adjuvant chemotherapy. We found patients were treated with neoadjuvant chemotherapy had statistically significant better OS compared to patients were treated with adjuvant chemotherapy (median OS was 48.6 months vs 38.6 months, $P<0.001$ ) as illustrated in the Kaplan-Meier survival curves

\section{Supplementary Files}

This is a list of supplementary files associated with this preprint. Click to download.

- SupplementaryFigureA.png

- SupplementaryFigureB.png

- SupplementaryFigureC.png

- SupplementaryFigureD.png

- SupplementaryFigureE.png

- SupplementaryFigureF.png

- SupplementaryFigureG.png

- SupplementarytableA.docx

- SupplementarytableB.docx 